\title{
Identification and prognosis analysis of germline WT1 (p.Arg370Pro) and TET2 (p.Asp1844Asn) mutations in an acute myeloid leukemia patient with $\mathrm{t}(9 ; 11)(\mathrm{p} 21.3 ; \mathrm{q} 23.3) ; K M 2 T A-M L L 3$
}

\section{Qiang Ma}

Xuanwu Hospital

Yixian Guo

Xuanwu Hospital

Xiaoxi Lan

Xuanwu Hospital

Guoxiang Wang

Xuanwu Hospital

Congyan Liu

Xuanwu Hospital

Jingjuan $\mathrm{He}$

Xuanwu Hospital

Xuejing Sun

Xuanwu Hospital

Yan Liu

Xuanwu Hospital

Suigui Wan

Xuanwu Hospital

Li Su

Xuanwu Hospital

Wanling Sun (D2965307416@qq.com)

Xuanwu Hospital

Research

Keywords: Acute myeloid leukemia, WT1,TET2, Germline mutation, Prognosis

Posted Date: December 20th, 2019

DOI: https://doi.org/10.21203/rs.2.19368/v1 
License: (c) (i) This work is licensed under a Creative Commons Attribution 4.0 International License. Read Full License 


\section{Abstract}

Background: Somatic mutations in WT1 and TET2 were separately perceived as contributors to hematopoietic disorders and thought to have a mutually exclusive effect in acute myeloid leukemia (AML). Here, we report a case in which WT1 and TET2 mutations co-existed at different stages in an AML patient with $\mathrm{t}(9 ; 11)(\mathrm{p} 21.3 ; \mathrm{q} 23.3)$, and without abnormal WT1 expression, which did not synchronize with the patient's tumor state. Hence, the origins and prognostic value of these two mutations were investigated.

Methods: Bone marrow (BM) and buccal mucosal cells were obtained from a 27-year-old male AML patient, and next-generation sequencing (NGS), targeting multiple genes, was performed after DNA extraction. Single nucleotide variant (SNV) and insertion-deletion (Indel) associated with AML were identified in these two samples by using SAM tools. Then, peripheral blood or buccal mucosal cells were obtained from the patient's relatives, and NGS targeting multiple genes was applied. Besides, the relationship between overall survival (OS) and alteration of these two genes in AML patients was analyzed by using cBioPortal and UALCAN databases, respectively. Results: SNV of WT1 (NM_024426:exon7:c.1109G>C囚p.Arg370Pro) and TET2 (NM_001127208:exon11:c.5530G>A】 p.Asp1844Asn) were present in the BM of the patient, and these two mutations were also observed in his buccal mucosal cells, which suggested that they were germline mutations. Variation analysis of samples from other relatives indicated that WT1 (NM_024426:exon7:c.1109G>C;p.Arg370Pro) and TET2 (NM_001127208:exon11:c.5530G>A;p.Asp1844Asn) were also present in his father and mother, respectively. The patient's brother also carried TET2 (NM_001127208:exon11:c.5530G>A囚p.Asp1844Asn) mutations. Bioinformatic analysis suggested that mutations in the WT1 and TET2 genes are both associated with a poor prognosis of AML, and abnormal TET2 expression is associated with a poorer prognosis than abnormal WT1 expression.

Conclusion: It is firstly report that WT1 p.Arg370Pro and TET2 p.Asp1844Asn mutations co-existed in an AML patient with $\mathrm{t}(9 ; 11)(\mathrm{p} 21.3 ; \mathrm{q} 23.3)$ and no WT1 abnormal expression, which highlight the importance of the identification and prognostic evaluation of germline mutations in clinical practice for AML.

\section{Background}

Acute myeloid leukemia (AML) is a clonal hematopoietic disorder and is usually observed along with genetic alterations in hematopoietic stem cells[1]. Most of the molecular cytogenetic abnormalities involved in AML have been identified, and these may be potential biomarkers for differential diagnosis, risk stratification, and therapeutic response in AML patients[2, 3]. Nevertheless, genetic alterations with definite clinical significance are relatively rare[4], and the clinical significance of most of them is still unknown[5], especially for germline mutations[6]. 
In our hospital, a 27-year-old male was diagnosed with AML, having $t(9 ; 11)(p 21.3 ; q 23.3) ;$ KM2TA-MLL3, accompanied by mutated Wilms' tumor 1 (WT1) and tet methylcytosine dioxygenase 2 (TET2), without abnormal WT1 mRNA expression. However, molecular genetic alterations were not consistent with the patient's tumor state. Hence, there are some noteworthy issues in this case, for instance: Why did WT1 and TET2 mutations, which were previously reported to be mutually exclusive[7], persistently co-exist in the AML patient, and do alterations in the TET2 and WT1 genes influence the overall survival of AML patients? In particular, which alterations are associated with poor prognosis-mutation or abnormal expression? The origin of two co-existing mutations was investigated in the patient's relatives, and their prognostic value was evaluated via two online databases to address these queries.

\section{Materials And Methods}

Case presentation

A 26-year-old male was admitted to our hospital in Jan 2019 due to hypoleukocytosis lasting two weeks. His family history did not suggest an inherited susceptibility to cancer, as no first-degree relatives, including his parents, his brother, and his child, had cancer. The complete blood cell count (CBC) indicated a white blood cell (WBC) count of $0.77 \times 10^{9} / \mathrm{L}$, platelet count of $129 \times 10^{9} / \mathrm{L}$, and hemoglobin $\mathrm{Hb})$ concentration of $45 \mathrm{~g} / \mathrm{L}$. Bone marrow aspirates revealed monocyte hyperplasia with $93 \%$ monoblasts and $2.5 \%$ promonocytes (Fig. 1A). Moreover, non-specific esterase stain revealed intensely positive monoblasts, and the positive stain could be inhibited by sodium fluoride (data not shown). Immunophenotype analysis of the abnormal cells by flow cytometry revealed full expression of HLA-DR, CD38, CD33, CD15, CD64 and CD56; partial expression of CD34, CD117, CD123, CD11b; and no expression of CD13, CD7, CD5, CD19, CD10, CD20, CD14, MP0 and CCD3 (Fig. 1C). Chromosome analysis of bone marrow cells indicated a karyotype of $46, \mathrm{XY}, \mathrm{t}(9 ; 11)(\mathrm{p} 21.3 ; q 23.3)$ (Fig. 1D). The KM2TA-MLL3 fusion gene was identified using PCR. Targeted multi-genes panel sequencing revealed that WT1 (p.Arg370Pro, VAF 49.19\%) and TET2 (p.Asp1844Asn, VAF 25.25\%) mutations were present. He was diagnosed with AML, having $\mathrm{t}(9 ; 11)(\mathrm{p} 21.3 ; \mathrm{q} 23.3)$, KM2TA-MLL3, accompanied by mutated WT1 and TET2, intermediate risk. A standard IA induction chemotherapy regimen (idarubicin + cytarabine) was administered on January 30, 2019, but bone marrow depression occurred.

At the end of first induction chemotherapy on February 20, 2019, the CBC revealed a WBC count of $0.64 \times$ $10^{9} / \mathrm{L}, \mathrm{Hb}$ of $83 \mathrm{~g} / \mathrm{L}$, platelet count of $21 \times 10^{9} / \mathrm{L}$. Bone marrow aspiration showed that primitive and immature cells made up $6 \%$ of the nucleated bone marrow cells. Flow cytometry detected $21 \%$ abnormal immunophenotype monocytes. The patient achieved partial remission. The CAG (Clarithromycin + Cytarabine + G-CSF) regimen was then administered as second induction chemotherapy on February 21, 2019.

The evaluation of the bone marrow at the end of second induction on March 21, 2019, showed that primitive and immature monocytes made up $0 \%$ of the bone marrow nucleated cells. Monocytes with abnormal immunophenotype were less than $0.01 \%$. The KM2TA-MLL3 fusion gene was not present. 
Complete remission was achieved. However, the WT1 (p.Arg370Pro VAF 50.11\%) and TET2 (p.Asp1844Asn VAF 48.33\%) mutations were still present. Another IA regimen was then administered as consolidation chemotherapy on March 29, 2019. The evaluation of the bone marrow on May 9, 2019 revealed that primitive and immature monocytes made up $7 \%$ of the nucleated bone marrow cells and CD34 + CD $117+$ CD33 + cells were $0.75 \%$ by flow cytometry. MLL3-AF9 fusion remained negative. All these data suggested early recurrence. While WT1 (p.Arg370Pro) and TET2 (p.Asp1844Asn) mutations were still positive, the VAF was $51.87 \%$ and $51.78 \%$, respectively.

Owing to the fluctuation of the disease, the risk stratification was re-evaluated to high-risk. A HA regimen chemotherapy (homoharringtonine and intermediate-dose cytarabine) was administered on May 11, 2019. The second complete remission was achieved, with mutations on WT1 (p.Arg370Pro) and TET2 (p.Asp1844Asn) genes still present, and the VAF was $48.35 \%$ and $49.88 \%$, respectively.

A sequential allogeneic HLA-matched single hematopoietic stem cell transplantation was carried out on July 9, 2019. Complete donor chimerism was received after allo-HSCT (Fig. 1E). To date, the patient has remained in complete remission with regular follow-up.

Since the patient's bone marrow-targeted multi-gene panel sequencing results did not synchronize with the patient's tumor stage, we suspected the WT1 (p.Arg370Pro) and TET2 (p.Asp1844Asn) mutations were germline mutations. To verify this hypothesis, we sampled the buccal mucosa cells of the patient and the peripheral blood or buccal mucosa cells of the patient's parents and brother.

DNA extraction and targeted multi-gene panel sequencing

DNA was extracted from the above samples according to the manufacturer's instructions (BioTeke Corporation, Beijing, China), and stored at $-20^{\circ} \mathrm{C}$. This study was approved by the Ethics Committee of Xuanwu Hospital, and written informed consent was obtained from all subjects. Variations of DNA samples from the patient's relatives were analyzed using NGS, based on a panel of thirty-four genes commonly involved in myeloid neoplasms (ASXL1, BCOR, BCORL1, CALR, CBL, CSF3R, IDH1, IDH2, JAK2, KRAS, MPL, NRAS, SF3B1, SH2B3, SRSF2, TET2, TP53, U2AF1, EZH2, SETBP1, ETV6, DNMT3A, ZRSR2, PHF6, FLT3, RUNX1, CEBPA, PIGA, WT1, PDGFRA, KIT, NPM1, GATA2, MLL). A PCR-based method was used for target-enrichment (KAPA Library Amplification Kits, Roche Diagnostics Corporation, Indianapolis, USA), and average sequencing depth was above 1500×. Variants were identified by SAMtools software, and variant allele frequency (VAF) was calculated as mutation reads/coverage reads[8].

Bioinformatics analysis

On the cBioPortal cancer genomics database (Version 3.1.0; https://www.cbioportal.org/,version 3.0.6)[9, 10], we accessed three studies including 1661 AML patients; two Acute Myeloid Leukemia (OHSU, Nature 2018 and TCGA, PanCancer Atlas), and one Pediatric Acute Myeloid Leukemia (TARGET, 2018). The alteration characteristics and prognostic value of WT1 and TET2 were analyzed via the cBioPortal 
database. Another database, UALCAN (http://ualcan.path.uab.edu/index.html) database[11], was used to analyze WT1/TET2 expression levels and their relationship with overall survival (OS) in AML patients.

\section{Results}

WT1 Arg370Pro and TET2 Asp1844Asn co-existed in this AML patient by inheritance

Genetic alterations of the patient at different stages are shown in Table 1. In the first clinical diagnosis, WT1 (NM_024426:exon7:c.1109G > C;p.Arg370Pro) (VAF = 49.19\%) and TET2

(NM_001127208:exon11:c.5530G > A A]p.Asp1844Asn) (VAF = 25.25\%) mutations were detected in the patient's BM sample. Then we found that the same WT1 and TET2 mutations were persistent in the later stages. To determine whether the mutually exclusive WT1 and TET2 mutations were germline mutations, buccal mucosa cells (somatic control) were first obtained from the patient for targeted sequencing, and results revealed that shared variation sites of WT1 Arg370Pro(52.09\%) and TET2 Asp1844Asn $₫ 37.26 \%$ lexisted in buccal mucosa cells, indicating that WT1 Arg370Pro and TET2 Asp1844Asn were germline mutations.

Table 1

Molecular and cellular genetic alternation of patient at different disease stages

\begin{tabular}{|c|c|c|c|c|c|c|c|}
\hline \multirow[t]{2}{*}{ Examination } & \multicolumn{4}{|c|}{ Before allo-HSCT } & \multicolumn{3}{|c|}{ After allo-HSCT } \\
\hline & $\begin{array}{l}\text { Initial } \\
\text { Diagnosis }\end{array}$ & CR1 & Relapse & CR2 & 1 & 2 & 3 \\
\hline Karyotype & $\begin{array}{l}\mathrm{t}(9 ; 11) \\
\text { (p21.3;q23.3) }\end{array}$ & NA & - & NA & - & - & NA \\
\hline Fusion & KM2TA-MLL3 & - & - & NA & - & NA & NA \\
\hline \multirow[t]{2}{*}{$\begin{array}{l}\text { Mutations } \\
\text { (VAF \%) }\end{array}$} & $\begin{array}{l}\text { TET2 } \\
(25.25)\end{array}$ & TET2(48.33) & $\begin{array}{l}\text { TET2 } \\
(51.78)\end{array}$ & $\begin{array}{l}\text { TET2 } \\
(49.88)\end{array}$ & \multirow[t]{2}{*}{$\begin{array}{l}\text { TET2 } \\
\text { (48.58) }\end{array}$} & \multirow[t]{2}{*}{$\begin{array}{l}\text { TET2 } \\
(50.20)\end{array}$} & \multirow[t]{2}{*}{$\begin{array}{l}\text { TET2 } \\
(52.59)\end{array}$} \\
\hline & $\begin{array}{l}\text { WT1 } \\
(49.19)\end{array}$ & WT1(50.11) & $\begin{array}{l}\text { WT1 } \\
(51.87)\end{array}$ & $\begin{array}{l}\text { WT1 } \\
(48.35)\end{array}$ & & & \\
\hline $\begin{array}{l}\text { WT1 level } \\
(\%)\end{array}$ & 0 & NA & 0.03 & 0.15 & NA & NA & 0 \\
\hline $\begin{array}{l}\text {-:Mutation or } \\
\text { hematopoiet } \\
\text { inductive che } \\
\text { level }(\%)=\text { WT }\end{array}$ & $\begin{array}{l}\text { ion gene neg } \\
\text { tem cell trans } \\
\text { therapy; CR2 } \\
\text { RRNA copies/ }\end{array}$ & $\begin{array}{l}+ \text { +:Mutation o } \\
\text { ation ; NA: Nc } \\
\text { plete remissi } \\
\text { mRNA copie }\end{array}$ & $\begin{array}{l}\text { Ision gen } \\
\text { vailable; } \\
\text { fter one }\end{array}$ & $\begin{array}{l}\text { positive; } \\
\text { 21: Comp } \\
\text { cle reinc }\end{array}$ & $\begin{array}{l}\text { Ilo-HSCT: } \\
\text { ete remiss } \\
\text { ctive cher }\end{array}$ & $\begin{array}{l}\text { Allogeneic } \\
\text { on after tv } \\
\text { lotherapy; }\end{array}$ & $\begin{array}{l}\text { cylces } \\
\text { VT1 }\end{array}$ \\
\hline
\end{tabular}

To further identify whether these two mutations were heritable, DNA samples from the patient's relatives were analyzed using the same target sequencing; WT1 Arg370Pro (VAF 44.75\%) was detected in the patient's father and TET2 Asp1844Asn (VAF 36.01\%) was detected in his mother; the brother carried the TET2 Asp1844Asn mutation (VAF 48.30\%) (Fig. 2). The above results further confirmed that germline 
mutations WT1 Arg370Pro and TET2 Asp1844Asn co-existed in the patient with acute monoblastic/monocytic leukemia.

WT1 and TET2 mutations, rather than WT1 expression, are both associated with poor prognosis of AML

In the initial diagnosis, WT1 mRNA expression level was undetectable in the BM of the patient.

Subsequently, although very low WT1 mRNA levels were observed at relapse and in second complete remission,they remained in the normal reference range. After allo-HSCT, WT1 mRNA levels were again elevated, but no abnormal expression was observed. These results indicate that WT1 mRNA expression did not reflect the change in the disease (Table 1).

The prognostic value of the expression levels and mutations of WT1 and TET2 were therefore explored. The OS of patients with or without WT1 and TET2 alteration was compared using the cBioPortal database; AML patients with WT1 or TET2 mutation were found to usually have a shorter OS than those without [median (18.42 vs 55 months) and (11.41 vs 57 months); $\mathrm{P}=3.108 \mathrm{e}^{-3}, \mathrm{P}=4.19 \mathrm{e}^{-10}$, respectively; Fig. $3 A$ and $B]$. Then, the prognostic value of WT1 and TET2 expression levels was analyzed via the UALCAN database. According to the median expression level of WT1 and TET2, patients with AML were divided into a high WT1 expression group $(n=42)$ and a low/medium WT1 expression group $(n=121)$, or a high TET2 expression group $(n=44)$ and a low/medium TET2 expression group $(n=119)$. No obvious association was noted between WT1 expression and prognosis $(P=0.43$; Fig. $3 C)$, but patients with TET2 high expression usually had a good prognosis $(P=0.0091$; Fig. 3D).

\section{Discussion}

In this study, we first reported that WT1 Arg370Pro co-exists with TET2 Asp1844Asn mutation in an AML patient, but without abnormal WT1 expression at different disease stages. These two mutations were derived from the patient's father and mother, respectively. In combination with the database analysis results, we determined that WT1 and TET2 mutations are both associated with poor prognosis in AML patients. However, the effect of expression level on prognosis differs. AML patients with TET2 high expression levels usually have a longer OS, but no significant difference was observed in AML patients with high and low/medium WT1 expression.

The relationship between WT1 and TET2 mutations was analyzed in the cBioportal database, and the results revealed that WT1 and TET2 mutations were mutually exclusive in AML patients $\left(P=0.011, \log _{2}\right.$ Odds Ratio - 2.160). Hence, it is rare that WT1 and TET2 mutations co-exist in an AML patient[12]. As the co-existing mutations in this case were germline mutations derived from the patient's father and mother, respectively, this case serves as a reminder that mutually exclusive germline mutations can co-exist in a patient due to inheritance. However, the role of germline mutations in a patient with AML was previously underappreciated. Recently, a growing number of germline mutations associated with tumor susceptibility have been reported; for instance, MITF (Microphthalmia-associated transcription factor) mutation impairs SUMOylation, which has an oncogenic function in the tumorigenesis of multiple 
tissues/melanocytes and kidney cells[13]. GATA2, RUNX1, DDX41, PAX5, CEBPA, and TP53 are associated with hematologic malignancies predisposition ${ }^{14}$. Identifying germline mutations is beneficial for therapy choice, donor selection for hematopoietic stem cell transplantation, evaluation of comorbidities, and surveillance strategies to improve the clinical outcomes[14]. In this case, after identifying germline mutations, we realized that it was inappropriate to use WT1 and TET2 mutations as indicators for minimal residual disease (MRD) monitoring. As for the treatment, although guidelines associated with germline mutations are limited, it is worth considering whether the risk stratification should be re-elevated and whether it is appropriate to start donor selection for allogeneic hematopoietic stem cell transplantation.

TET2 is important for normal myelopoiesis, and disruption of TET2 enzymatic activity favors myeloid tumorigenesis[15]. Slower proliferation and reduced clonogenicity have been observed in TET2 overexpressing cells in vitro compared to those transfected with the empty vector, which indicates the suppressor role of TET2 in AML. However, the inhibitory effects of TET2 on leukemic cell proliferation and colony formation could be abolished by WT1 knockdown ${ }^{[15]}$. Further studies revealed that TET2 suppresses leukemia cell proliferation and colony formation in a WT1-dependent manner[16]. All of the above conclusions are from in vitro and animal experiments; here, for the first time, TET2 and WT1 mutations are reported to co-exist in an AML patient, with detectable alterations only in the complete remission and relapse stages. From this, we must consider whether co-existing WT1 and TET2 mutations lead to the occurrence and progression of $A M L$ in the above manner.

Since the interaction of these two genes is essential for biological functions, we attempted to explain the pathogenesis at the molecular level. TET2 binds to the zinc finger domain (residue 323-449), but not the $\mathrm{N}$-terminal region (residue 1-323) of WT1. In this patient, the missense mutation was in the zinc finger domain (residue 370) of WT1, which may disrupt the binding between WT1 and TET2. Moreover, WT1 binds to the CD domain of TET2, but not the N-terminal region (residue 1 to 1127)[15]. Unfortunately, in this case, the missense mutation was in the CD domain (residue 1844) of TET2, which may further disrupt the binding between WT1 and TET2. From a molecular point of view, this may be a reasonable explanation for the disease. In clinical practice, WT1 expression and disease progression are usually parallel to each other; WT1 expression is also used as the MRD marker of hematological neoplasms when other genetic alterations are absent[17]. However, in this case, abnormal up-regulation of WT1 was not observed at different stages, but the WT1 mutation persisted. Similar to the WT1 mutation being related to poor prognosis[18], WT1 overexpression was also indicated to be associated with shorter OS[19]. However, the question remains, which alteration could be a potential indicator of prognostic stratification for the patient; expression level or mutation status? It seems contradictory for the patient to have WT1 mutation and no WT1 overexpression, and related reports in the literature are limited. Here, analysis results based on special tumor databases suggested that the alteration frequency and expression level of the WT1 gene was different in various AML subtypes. Among them, acute monoblastic/monocytic leukemia has the highest WT1 mutation frequency and the lowest mRNA levels, which may explain why the patient had WT1 mutation but not WT1 overexpression(data not shown). 
In this case, WT1 and TET2 mutations were the only molecular alterations in the relapse stage, and whether the co-existence of WT1 and TET2 was the cause of the relapse was unknown. However, previous studies have revealed that WT1 can bind TET2, acting as a guide for TET2 to specific genomic loci, leading to increased stem cell function and self-renewal with proliferation[20]. This seems a reasonable explanation for why the patient relapsed in a short time, but studies on the effect of WT1 and TET2 co-existence on AML occurrence and development are limited. It is not easy to obtain direct evidence that WT1 and TET2 co-existence was the cause of relapse AML, but the OS of AML with WT1 and/or TET2 alteration could be investigated via tumor databases. Here, we found that the alteration of WT1 or TET2 is associated with a poor prognosis. Patients with WT1 or/and TET2 mutations usually have a worse prognosis than those without any alteration. Further, TET2 high expression is also associated with a good prognosis. However, contrary to previous studies[21], we observed no obvious correlation between WT1 mRNA expression and OS in AML patients. Although we found WT1 and TET2 mutations co-existed in an AML patient and explored the relationship between the alteration of these two genes and prognosis, there are still some limitations to this study. For example, other factors can influence the prognosis of $\mathrm{AML}$, and it is necessary to take them into account as well. Moreover, the issue of whether there are prognostic differences between somatic or germline mutations of the same genes should be investigated via further effective research.

\section{Conclusions}

We present the first report that mutually exclusive WT1 and TET2 mutations co-existed in an AML patient; these were identified as germline mutations. In particular, WT1 and TET2 mutations were both associated with a poor prognosis, but no prognostic value was observed for WT1 expression levels.

\section{Declarations}

\section{Acknowledgements}

Not applicable

\section{Authors' contributions}

QM performed this study and wrote the manuscript. YG provided case presentation, $\mathrm{XL}, \mathrm{GW}$ collected samples. CL, JH, XS, YL, SW, LS performed laboratory and clinical diagnosis. WS designed this study, critically reviewed the manuscript, and gave final approval.

\section{Funding}

No funding was received. 


\section{Availability of data and materials}

All data generated during this study are included in this published article.

\section{Consent for publication}

Not applicable

\section{Competing interests}

The authors declare that they have no competing interests.

\section{Ethics approval and consent to participate}

This study was approved by the Ethics Committee of Xuanwu Hospital, and written informed consent was obtained from all subjects.

\section{References}

1. Allahyari A, Tajeri T, Sadeghi M. Prognostic Factors and Survival in Acute Myeloid Leukemia Cases: a Report from the Northeast of Iran. Asian Pacific journal of cancer prevention : APJCP 2016;17:154751.

2. Iwabuchi S, Fukui S, Sugai J. [Post-transfusion acute non-A, non-B hepatitis]. Nihon rinsho Japanese journal of clinical medicine 1988;46:2663-8.

3. Vosberg S, Greif PA. Clonal evolution of acute myeloid leukemia from diagnosis to relapse. Genes, chromosomes \& cancer 2019.

4. Wang M, Yang C, Zhang L, Schaar DG. Molecular Mutations and Their Cooccurrences in Cytogenetically Normal Acute Myeloid Leukemia. Stem cells international 2017;2017:6962379.

5. Hirsch P, Qassa G, Marzac C, et al. Acute myeloid leukemia in patients older than 75: prognostic impact of FLT3-ITD and NPM1 mutations. Leukemia \& lymphoma 2015;56:147-50.

6. Maciejewski JP, Padgett RA, Brown AL, Muller-Tidow C. DDX41-related myeloid neoplasia. Seminars in hematology 2017;54:94-7. 
7. Du D, Zhu L, Wang Y, YeX. [Expression of WT1 gene and its prognostic value in patients with acute myeloid leukemia]. Zhejiang da xue xue bao Yi xue ban = Journal of Zhejiang University Medical sciences 2019;48:50-7.

8. Young AL, Wong TN, Hughes AE, et al. Quantifying ultra-rare pre-leukemic clones via targeted errorcorrected sequencing. Leukemia 2015;29:1608-11.

9. Gao J, Aksoy BA, Dogrusoz U, et al. Integrative analysis of complex cancer genomics and clinical profiles using the cBioPortal. Science signaling 2013;6:pl1.

10. Cerami E, Gao J, Dogrusoz U, et al. The cBio cancer genomics portal: an open platform for exploring multidimensional cancer genomics data. Cancer discovery 2012;2:401-4.

11. Chandrashekar DS, Bashel B, Balasubramanya SAH, et al. UALCAN: A Portal for Facilitating Tumor Subgroup Gene Expression and Survival Analyses. Neoplasia 2017;19:649-58.

12. Bowman RL, Levine RL. TET2 in Normal and Malignant Hematopoiesis. Cold Spring Harbor perspectives in medicine 2017;7.

13. Paillerets BB, Lesueur F, Bertolotto C. A germline oncogenic MITF mutation and tumor susceptibility. European journal of cell biology 2014;93:71-5.

14. Furutani E, Shimamura A. Germline Genetic Predisposition to Hematologic Malignancy. Journal of clinical oncology : official journal of the American Society of Clinical Oncology 2017;35:1018-28.

15. Ko M, Huang Y, Jankowska AM, et al. Impaired hydroxylation of 5-methylcytosine in myeloid cancers with mutant TET2. Nature 2010;468:839-43.

16. Wang $Y$, Xiao $M$, Chen $X$, et al. WT1 recruits TET2 to regulate its target gene expression and suppress leukemia cell proliferation. Molecular cell 2015;57:662-73.

17. Rossi G, Minervini MM, Carella AM, Melillo L, Cascavilla N. Wilms' Tumor Gene (WT1) Expression and Minimal Residual Disease in Acute Myeloid Leukemia. In: van den Heuvel-Eibrink MM, ed. Wilms Tumor. Brisbane (AU) 2016.

18. Niktoreh N, Walter C, Zimmermann M, et al. Mutated WT1, FLT3-ITD, and NUP98-NSD1 Fusion in Various Combinations Define a Poor Prognostic Group in Pediatric Acute Myeloid Leukemia. Journal of oncology 2019;2019:1609128.

19. Liu H, Wang $X$, Zhang $H$, et al. Dynamic changes in the level of WT1 as an MRD marker to predict the therapeutic outcome of patients with AML with and without allogeneic stem cell transplantation. Molecular medicine reports 2019;20:2426-32.

20. Pronier E, Bowman RL, Ahn J, et al. Genetic and epigenetic evolution as a contributor to WT1-mutant leukemogenesis. Blood 2018;132:1265-78.

21. Hu ZL, Huang BJ, Pan SJ. [Effect of WT1 Gene High Expression and CD34(+) and Auer(+) on Prognosis of Acute Myeloid Leukemia]. Zhongguo shi yan xue ye xue za zhi 2016;24:1670-4.

\section{Figures}


A

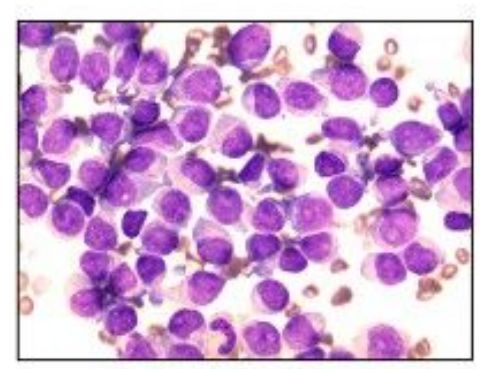

$\mathrm{C}$
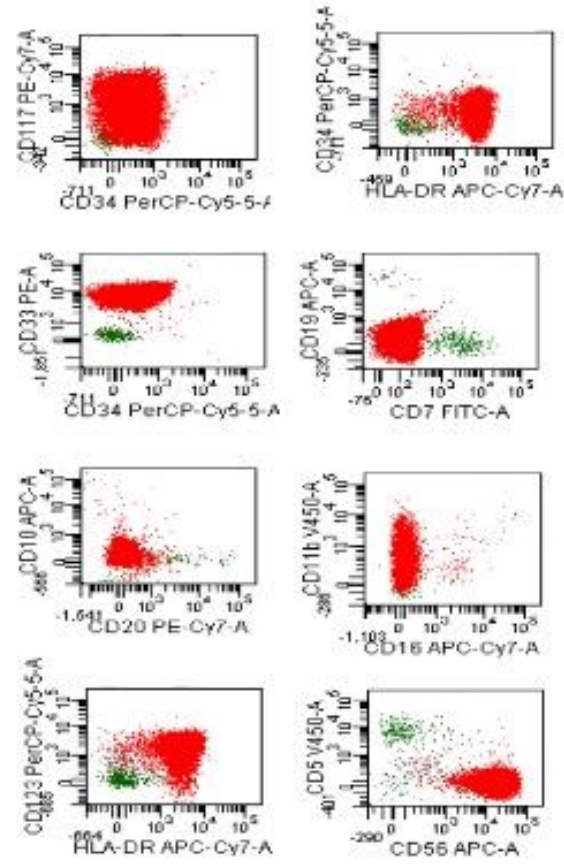

B
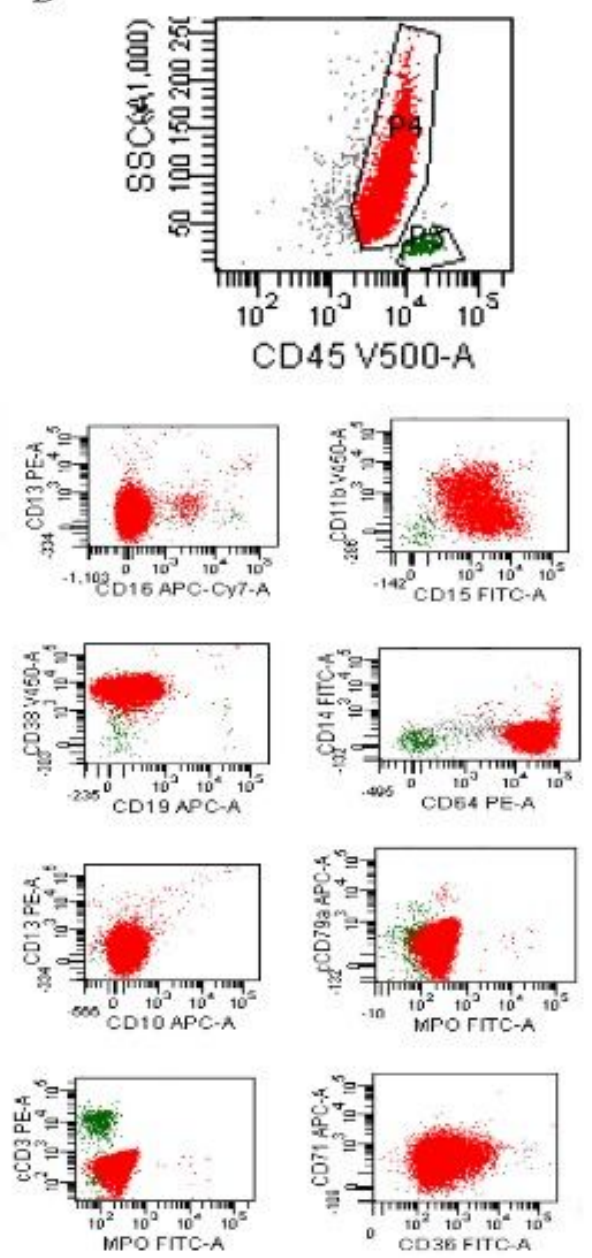

$\mathrm{E}$

\section{4 is

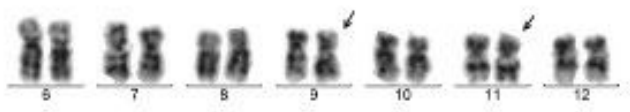

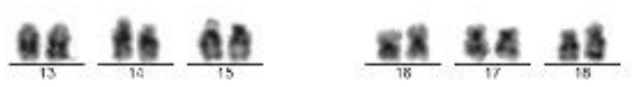

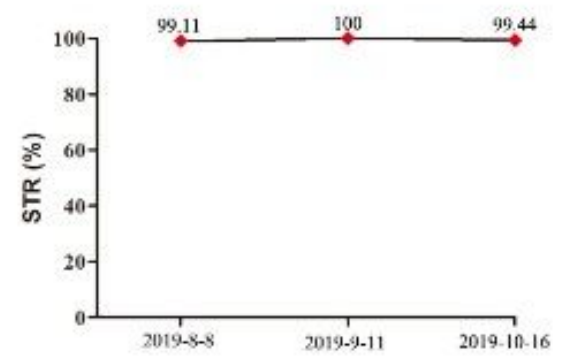

\section{Figure 2}

Representative results of laboratory tests of the patient with AML. (A) Monoblasts were observed in bone marrow of the patient, and some had very abundant cytoplasm; (B-C) P4 stands for abnormal cells, accounted for 96\%. HLA-DR(+), CD33(+), CD15(+), CD64(+), CD56(+), CD38(+), CD123(+), CD56(+);CD11b(partly +), CD117(partly +); CD10(-), CD19(-), CD20(-), MPO(-), cCD3(-), cCD79a(-), CD13(-), CD7(-), CD5(-), CD14(-). (D) Chromosome karyotype analysis showed that the patient with $46, \mathrm{XY}, \mathrm{t}(9 ; 11)$ 
(p21.3;q23.3). (E) Complete donor chimerism was observed after allo-HSCT by short tandem repeat polymerase chain reaction (STR-PCR) analysis.

娄

\section{Figure 4}

Genetic change of WT1 and TET2 in patient's relatives. Mutation status and VAF value of WT1/TET2 in the patient's relatives.

\section{Figure 6}

The association between OS and WT1/TET2 genes. (A) Prognostic comparison of patients with or without WT1 alteration; (B) Prognostic comparison of patients with or without TET2 alteration; (C) The OS of AML patients with high WT1 expression and with low/medium expression; (D) The OS of AML patients with high TET2 expression and with low/medium expression. 